\title{
Solitary Plasmacytoma
}

National Cancer Institute

\section{Source}

National Cancer Institute. Solitary Plasmacytoma. NCI Thesaurus. Code C6932.

A localized clonal (malignant) plasma cell infiltrate either in the bony skeleton or soft tissue without peripheral blood involvement. 